\title{
Erster Nachweis der Solitärbienenart Colletes halophilus Verhoeff, 1944 in Schleswig-Holstein (Hymenoptera: Colletidae)
}

\author{
First record of the solitary bee species Colletes halophilus Verhoeff, \\ 1944 in Schleswig-Holstein (Hymenoptera: Colletidae)
}

\author{
Jorinde Klammer, Monika Povel, Michael Kuhlmann \\ Zoologisches Museum der Christian-Albrechts-Universität zu Kiel, Hegewischstraße 3, 24105 Kiel, \\ mkuhlmann@zoolmuseum.uni-kiel.de
}

\section{Schlüsselwörter: \\ Erstnachweis, Solitärbiene, Verbreitung, Küstenhabitat, Salzwiese, Aster tripolium}

Keywords:

First record, solitary bee, distribution, costal habitat, salt marsh, Aster tripolium

\begin{abstract}
Zusammenfassung
Die an der Küste endemische Seidenbienenart Colletes halophilus Verhoeff, 1944 konnte 2015 erstmals am Deich der Eidermündung (Nordfriesland) für Schleswig-Holstein nachgewiesen werden. In 2017 konnte zusätzlich in Salzwiesen vor St. Peter-Ording eine Population ausfindig gemacht werden. Bisher gab es für Deutschland lediglich Funde an der Küste Niedersachsens und den vorgelagerten Inseln. Die Neufunde werden im Kontext der Gesamtverbreitung von C. halophilus und dem Vorkommen ihrer bevorzugten Pollenquelle, der Strandaster (Aster tripolium), diskutiert.
\end{abstract}

\begin{abstract}
The costal endemic solitary bee species Colletes halophilus Verhoeff, 1944 was first recorded for Schleswig-Holstein in 2015 at the sea dyke at the mouth of river Eider (North Frisia). In 2017 an additional population was discovered in a salt marsh close to St. Peter-Ording. So far in Germany the species was only known from the coast and islands of Lower Saxony. The new records are discussed in the context of the species' range and the distribution of its preferred pollen source, the Sea Aster (Aster tripolium).
\end{abstract}

\section{Einleitung}

Von den 582 in Deutschland vorkommenden Wild-bienenarten (Scheuchl \& Schwenninger 2015), konnten in Schleswig-Holstein bislang 296 Arten (Smissen 2001) nachgewiesen werden. Deutschlandweit werden 24 Wildbienenarten als „extrem selten“ eingestuft, darunter auch die Küsten-Seidenbiene Colletes halophilus Verhoeff, 1944 (Abb. 1) (Westrich et al 2011). Für diese Art hat Deutschland aufgrund seines großen Anteils am Gesamtareal eine besondere Verantwortlichkeit (Kuhlmann 2005).

Die Küsten-Seidenbiene gehört zur Colletes succinctusGruppe, zu der auch die Arten $C$. hederae Schmidt \&
Westrich, 1993 und C. succinctus Linnaeus, 1758 gehören. Die Arten sind anhand morphologischer Merkmale nur schwer zu trennen und genetisch sehr nah verwandt, unterscheiden sich aber in ihren Habitatpräferenzen, Phänologie und Blütenspezialisierung (Kuhlmann et al. 2007). Colletes halophilus ist mit Küstendünen und Salzwiesen assoziiert und fliegt von Anfang August bis Ende September. Die Art ist oligolektisch und spezialisiert auf Korbblütler (Asteraceae), wobei der Hauptanteil der Larvennahrung aus der Strandaster (Aster tripolium) besteht (Sommeijer et al. 2009). Die solitäre Wildbienenart nistet im Boden, in sandigen, vertikalen wie auch horizontalen Bereichen (Hardy 2013). Als Brutparasiten von 
C. halophilus sind die Kuckucksbienen Epeolus variegatus Linnaeus, 1758 (Guichard 1974) und im Südwesten der Niederlande Epeolus tarsalis Morawitz, 1874 beschrieben worden (Peeters et al. 1999). Bisher war C. halophilus in Deutschland nur aus Niedersachsen bekannt (Kuhlmann et al. 2007). Im Folgenden werden die ersten Nachweise von $C$. halophilus in SchleswigHolstein beschrieben und im Kontext der Gesamtverbreitung der Art sowie dem Vorkommen ihrer bevorzugten Pollenquelle, der Strandaster (Aster tripolium), diskutiert.

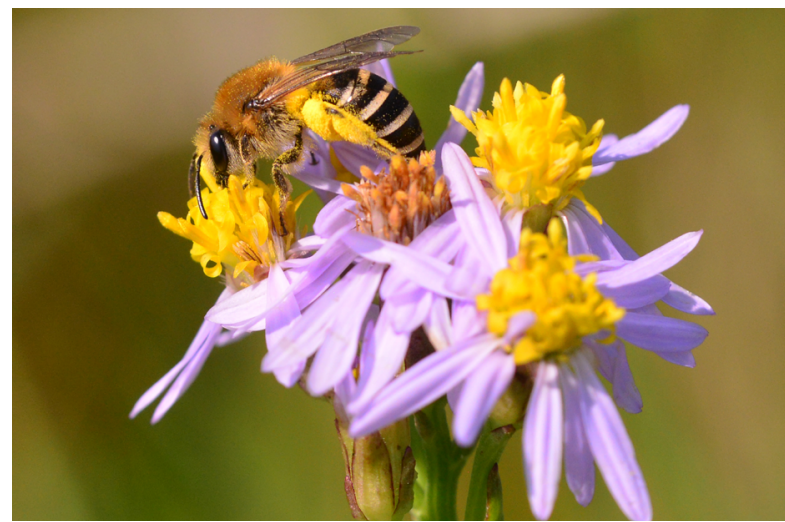

Abb. 1: Weibchen der Küsten-Seidenbiene (Colletes halophilus) an ihrer bevorzugten Pollenquelle, der Strandaster (Aster tripolium) (Foto: Monika Povel).

\section{Colletes halophilus in Schleswig- Holstein}

Bereits in den Jahren 2015 und 2016 wurde von einem von uns (MP) an der Eidermündung im Bereich des Seedeichs eine Seidenbienen-Art beobachtet, bei der es sich vermutlich um $C$. halophilus handelte. Aber erst 2017 konnten dort Exemplare gefangen und die Identität bestätigt werden. Im selben Jahr wurde auf Verdacht zusätzlich in St. Peter-Ording eine Salzwiesenfläche mit zahlreichen blühenden Strandastern auf das Vorkommen der Art hin untersucht. Hier konnte $C$. halophilus ebenfalls festgestellt werden (Belegexemplare im Zoologischen Museum Kiel).

\section{Vorkommen am Seedeich (nördliche Eidermündung)}

Der Fundort befindet sich im südlichsten Bereich der Halbinsel Eiderstedt (Nordfriesland) nahe der Landkreisgrenze zu Dithmarschen, an der Eidermündung. Zur Nordsee hin wird das Festland von einem Deich be- grenzt. Der Deich besteht aus einer Teerdecke und weist zum Watt hin eine einbetonierte Steinschüttung auf. In der Steinschüttung sind im Lauf der Jahre Ritzen und offene Bereiche entstanden. An die Steinschüttung schließt sich ein schmaler Streifen Salzwiese von zwei bis zehn Metern Breite an, der sich seit der Fertigstellung des Deiches 1973 bis heute hin entwickelt hat (Abb. 2).

In den Jahren 2015 (22. August) und 2016 (23. August) wurden bereits an dem beschriebenen Abschnitt des Seedeichs Weibchen von C. halophilus an A. tripolium gesichtet. Außerdem wurden 2016 Weibchen beobachtet,

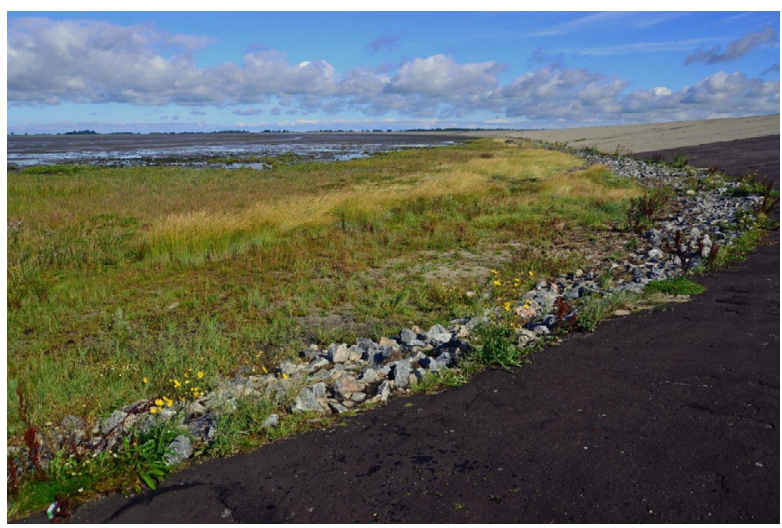

Abb. 2: Seedeich nördlich des Eidersperrwerks im Bereich des Vorkommens von Colletes halophilus mit angrenzender Steinschüttung und Salzwiese (Foto: Monika Povel).

die in Ritzen der Steinschüttung vermutlich ihre Nester anlegten, und Männchen, die in diesem Bereich patrouillierten. Am 14.8.2017 wurden die ersten Männchen von $C$. halophilus an A. tripolium ausgemacht und Belegexemplare zur Artbestimmung gefangen (E08 ${ }^{\circ} 50^{\prime} 50^{\prime \prime}$ N54 $16^{\prime} 08^{\prime \prime}$, leg. M. Povel, det. M. Kuhlmann). Vier Tage später erschienen die ersten Weibchen in der Nähe an der Steinschüttung des Deiches. Dort gesammelte Belegexemplare erwiesen sich ebenfalls als $C$. halophilus (E0850'40" N54²16'11", leg. M. Povel, det. M. Kuhlmann). Mutmaßliche Nester befanden sich an zwei offenen, sandigen Stellen in der Salzwiese. Da hier keine Individuen gesehen wurden, kann nicht mit Sicherheit gesagt werden, ob diese von $C$. halophilus stammten. Jedoch sammelten in unmittelbarer Nähe zwei Weibchen an A. tripolium Pollen und Nektar.

\section{Vorkommen bei St. Peter-Ording}

St. Peter-Ording befindet sich an dem westlichen Ende der Halbinsel Eiderstedt. Ohne künstliche Maßnah- 
men entstand hier im Schutz der $8 \mathrm{~km}$ langen Sandbank und Vordünen ein bis zu $1 \mathrm{~km}$ breites Vorland, welches zum Nationalpark Schleswig-Holsteinisches Wattenmeer gehört. Hierbei handelt es sich um SandSalzmarschen, in Schleswig-Holstein nur noch von Sylt, Amrum und Trischen bekannt (Gettner 2011). Der Bereich der Salzwiese, in der $C$. halophilus beobachtet wurde, befindet sich am Südstrand vor dem Ortsteil Dorf (Abb. 3). Vor der Salzwiese befindet sich zum Deich hin ein $10 \mathrm{~m}$ breiter aufgeschütteter Sandstrand, welcher touristisch genutzt wird. Daran grenzt die Salzwiese mit einer Breite von ungefähr 15 m (Gettner 2011). Eine detaillierte Beschreibung der vorhandenen Vegetation ist bei Rohman (2011) zu finden.

Am 23.8.2017 sammelten hier schätzungsweise 30 Individuen von $C$. halophilus Pollen und Nektar an Strandastern. Es konnten keine Nester ausfindig gemacht werden. Zwei Weibchen wurden als Belegexemplare entnommen (E08 $37^{\prime} 25^{\prime \prime}$ N541' $14^{\prime}$ ", leg. M. Povel, det. M. Kuhlmann).

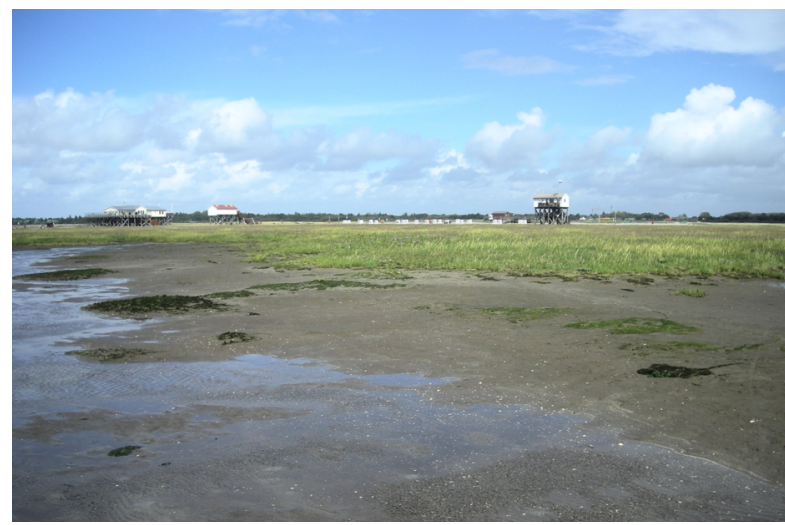

Abb. 3: Salzwiese mit Vorkommen von Colletes halophilus bei St Peter-Ording vor dem Ortsteil Dorf mit angrenzender Wattfläche. Auf der Höhe des rechten Pfahlbaus befindet sich der Sandstrand (Foto: Jorinde Klammer).

\section{Diskussion}

Das bislang bekannte Gesamtverbreitungsgebiet von $C$ halophilus erstreckt sich entlang der Atlantikküste Frankreichs, Belgiens, der Niederlande, Deutschlands und Südost-Englands (Kuhlmann et al. 2007, Genoud \& Dittlo 2007, Gilles 2009). Der bisher nördlichste Nachweis wurde erst kürzlich in Dänemark auf der Insel Fanø in Sønderho erbracht (Madsen et al. 2015). In Deutschland war die Art nur von der Küste Niedersachsens und den vorgelagerten Inseln bekannt (zusammengefasst in
Kuhlmann et al. 2007). Die neuen Funde in SchleswigHolstein auf der Halbinsel Eiderstedt sind die nördlichsten Nachweise der Art in Deutschland und schließen die Lücke zwischen den niedersächsischen und dem nördlichsten Vorkommen in Dänemark (Abb. 4).

Die jüngsten Neunachweise von $C$. halophilus sind die nördlichsten Funde der Art in Dänemark und in Schleswig-Holstein. Es ist wahrscheinlich, dass es sich hier um eine Neubesiedlung und damit Arealerweiterung handelt, da gerade die Westküste Schleswig-Holsteins seit Jahren immer wieder intensiv auf Wildbienen untersucht wurde (Haeseler, pers. Mitt.). Die Nordausbreitung von C. halophilus könnte eine Folge der auch für Schleswig-Holstein nachgewiesenen Klimaerwärmung sein (DWD 2017), die in anderen Teilen Europas in ähnlicher Weise an Libellen (Hickling et al. 2005, Grewe et al. 2013) und Schmetterlingen (Parmesan et al. 1999) beobachtet worden ist.

Ein wichtiger limitierender Faktor bei der Ausbreitung von Bienenarten ist das Vorhandensein geeigneter Nistplätze (Westrich 1990). Bei C. halophilus werden

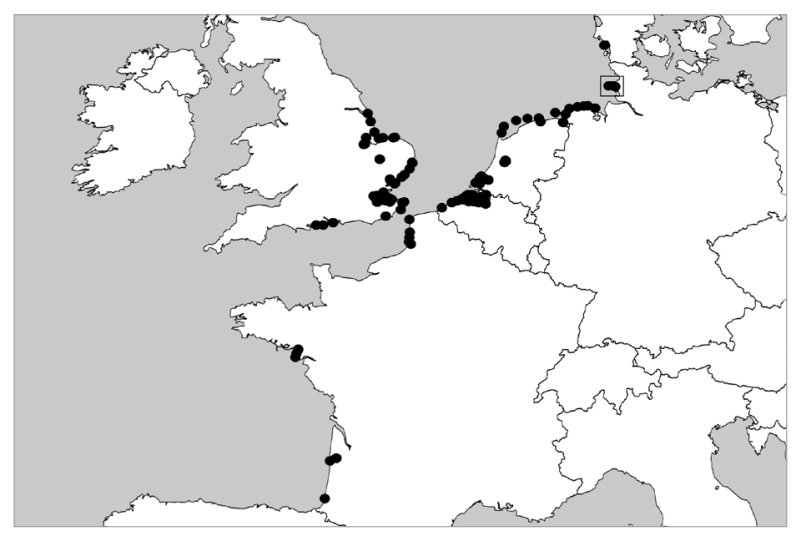

Abb. 4: Gesamtverbreitung von Colletes halophilus. Die umrahmten Punkte markieren die Neunachweise in Schleswig-Holstein.

die Brutzellen in lockeren Sand gebaut (Kuhlmann et al. 2007, Sommeijer et al. 2012). Aufgrund ihrer Präferenz für $A$. tripolium als Pollenquelle benötigt $C$. halophilus entsprechend räumlich eng verzahnte Vorkommen der Strandaster, z.B. Salzwiesen, und trockener offener, sandiger Bereiche für ihren Nestbau. Da die Strandaster entlang der schleswig-holsteinischen Westküste weit verbreitet ist, fehlt es vermutlich an geeigneten Nistplätzen in unmittelbarer Nachbar-schaft der Blüten, um die Region in größerem Umfang zu besiedeln. Zudem können Maßnahmen des Küstenschutzes durch Verbauungen geeignete Nisthabitate zerstören (Westrich 1990). Auch die 
Population am Deich nördlich des Eidersperrwerks ist vom Küstenschutz unmittelbar betroffen, da voraussichtlich 2020 hier mit der Erneuerung des Deichs begonnen wird (Schrader, pers. Mitt.). In St. Peter-Ording befindet sich das Vorkommen der Küsten-Seidenbiene im Nationalpark und ist vermutlich nicht direkt gefährdet.

Die Klimaerwärmung, die eine Nordwanderung von C. halophilus mutmaßlich erst ermöglicht hat, stellt gleichzeitig aber auch eine mögliche Gefährdung für bestehende Populationen dar. Mit dem prognostizierten (Vermeer \& Rahmstorf 2009) und in der Deutschen Bucht bereits beobachtbaren Anstieg des Meeresspiegels von deutlich über einem Meter bis Ende des 21. Jahrhunderts (DWD 2017), ist an den Küsten mit weitreichenden Veränderungen zu rechnen, etwa der Erosion von Nist- und Nahrungshabitaten, deren Auswirkungen auf die Küsten-Seidenbiene nicht vorhersehbar sind.

Um ein genaueres Bild der Verbreitung und Bestandssituation der Küsten-Seidenbiene in Schleswig-Holstein $\mathrm{zu}$ bekommen und um eine eventuelle Ausbreitung zu dokumentieren, sollten potentiell geeignete Habitate auf Vorkommen der Art untersucht und insbesondere die Nistbereiche erfasst werden. So ist aufgrund der Ausdehnung geeigneter Lebensräume im Bereich von St. PeterOrding zu vermuten, dass dort weitere, möglicherweise individuenreiche Populationen existieren. Sylt, Amrum und Trischen könnten aufgrund ihrer Sand-Salzmarschen (Gettner 2011) ebenfalls für C. halophilus geeignet sein.

\section{Autor*innen}

\section{Jorinde Klammer}

Jorinde Klammer arbeitet in der unteren Naturschutzbehörde im Kreis Ostholstein, unter anderem in dem Tätigkeitsbereich Artenschutz, Schutzgebiete und Naturschutzmaßnahmen. Während des Studiums der Biologie befasste sie sich insbesondere mit Wiesen als Lebensraum für die Überwinterung von Insekten und mit der Wildbienendiversität der Küstendünen von St. Peter-Ording.

\footnotetext{
Monika Povel

Monika Povel befasst sich seit 2011 als Nicht-Biologin und Hobby-Fotografin mit Stechimmen. Seit ihrem Anschluss an die Stechimmen AG der FÖAG erfasst sie Stechimmen vorallem in Nordfriesland (Bordelumer Heide, Dünen von St. Peter-Ording, Katinger Watt).
}

\section{Michael Kuhlmann}

Michael Kuhlmann ist Entomologe und Kurator am Zoologischen Museum der Christian-Albrechts-Universität zu Kiel. Sein Hauptarbeitsgebiet ist die Taxonomie, Verbreitung und Lebensweise von Wildbienen, speziell der Seidenbienen (Gattung Colletes). Weitere Forschungsfelder sind die Bienenfauna des südlichen Afrika sowie Blüte-Bestäuber-Beziehungen.

\section{Literatur}

DWD (2017) Klimareport Schleswig-Holstein. Deutscher Wetterdienst Offenbach am Main.

Genoud D, Dittlo F (2007) Contribution à la connaissance de Colletes halophilus Verhoeff (Hymenoptera, Colletidae) en France et confirmation de sa presence en Aquitaine. Osmia 1:3-4, doi: 10.47446/OSMIA1.1.

Gettner S (2011) Vegetationskundliche Untersuchungen im Vorland von St. Peter-Ording. Mitteilungen der AG Geobotanik in Schleswig-Holstein und Hamburg 67:89-168.

Gilles M (2009) Les abeilles du genre Colletes (Hymenoptera, Colletidae) en Presqu'ile guérandaise (Loire-Atlantique, France). Osmia 3:7-11.

Grewe Y, Hof C, Dehling DM, Brandl R, Brändle M (2013) Recent range shifts of European dragonflies provide support for an inverse relationship between habitat predictability and dispersal. Glob. Ecol. Biogeogr. 22:403-409.

Guichard KM (1974) Colletes halophila Verhoeff (Hymenoptera, Apidae) and its Epeolus parasite at Swanscombe in Kent, with a key to the British species of Colletes Latreille. Entomol.'s Gaz. 25:195-199.

Madsen HB, Schmidt HT, Bygebjerg R, Rasmussen C (2015) Tre nye arter af bier for den danske fauna (Hymenoptera, Apoidea). Entomologiske Meddelelser 83:21-29.

Hardy KA (2013) Investigation into the habitat requirements of the Sea Aster mining bee in both manmade and natural habitats: Implications for conservation management actions to improve habitat opportunities with a view to enabling the reconnection of isolated populations. Buglife, doi: 10.13140/2.1.3154.0482.

Hickling R, Roy DB, Hill JK, Thomas CD (2005) A northward shift of range margins in British Odonata. Global Change Biol. 11:502-506.

Kuhlmann M (2005) Vorläufige Einschätzung der Verantwortlichkeit Deutschlands für die Erhaltung von Stechimmenarten. In: Gruttke, H. (Hg.): Ermittlung der Verantwortlichkeit für die Erhaltung mitteleuropäischer Arten. Naturschutz und Biologische Vielfalt 8:225-233.

Kuhlmann M, Else GR, Dawson A, Quicke DL (2007) Molecular, biogeographical and phenological evidence for the existence of three western European sibling species in the Colletes succinctus group (Hymenoptera: Apidae). Org. Divers. Evol. 7:155-165.

Parmesan C, Ryrholm N, Stefanescu C, Hill JK, Thomas CD, Descimon H, Huntley B, Kaila L, Kullberg J, Tammaru T, Tennent WJ, Thomas JA, Warren M (1999) Poleward shifts in geographical ranges of butterfly species associated with regional warming. Nature 399:579-583.

Peeters TM, Raemakers IP, Smit J (1999) Voorlopige atlas van de Nederlandse bijen (Apidae). EIS-Nederland, Leiden.

Romahn K, Gettner S, Rennekamp G, Kieckbusch JJ (2011) Die Küstenlandschaft von St. Peter-Ording - ein Hotspot der Artenvielfalt. Mitteilungen $\operatorname{der}$ AG Geobotanik in Schleswig-Holstein und Hamburg 67:7-87.

Scheuchl E, Schwenninger HR (2015) Kritisches Verzeichnis und aktuelle 
Checkliste der Wildbienen Deutschlands (Hymenoptera, Anthophila) sowie Anmerkungen zur Gefährdung. Mitteilungen des Entomologischen Vereins Stuttgart 50:3-226.

Smissen J van der (2001) Die Wildbienen und Wespen Schleswig-Holsteins - Rote Liste. Band I-III. Landesamt für Natur und Umwelt des Landes Schleswig-Holstein, Flintbek.

Sommeijer MJ, Neve J, Jacobusse C (2012) The typical development cycle of the solitary bee Colletes halophilus. Entomologische Berichten 72:52-58.

Sommeijer MJ, Rooijakkers EF, Jacobusse C, Kerkvliet JD (2009) Larval food composition and food plants of the solitary bee Colletes halophilus (Hymenoptera: Colletidae). J. Apic. Res. 48:149-155.

Vermeer M, Rahmstorf S (2009) Global sea level linked to global temperature. PNAS 106:21527-21532, doi: 10.1073/pnas.0907765106.

Westrich P (1995) Die Wildbienen Baden-Württembergs - Allgemeiner Teil: Lebensräume, Verhalten, Ökologie und Schutz, 2. Auflage. Eugen Ulmer, Stuttgart.

Westrich P, Frommer U, Mandery K, Riemann H, Ruhnke H, Saure C, Voith $J(2011)$ Rote Liste und Gesamtartenliste der Bienen (Hymenoptera, Apidae) Deutschlands. Naturschutz und Biologische Vielfalt 70:373-416.

\section{Open Access}

> Der Artikel ist unter der Creative-Commons-Lizenz Namensnennung 4.0 International veröffentlicht. Den Vertragstext finden Sie unter: https://creativecommons.org/licenses/by/4.0/deed.de. Bitte beachten Sie, dass einzelne, entsprechend gekennzeichnete Teile des Artikels von der genannten Lizenz ausgenommen sein bzw. anderen urheberrechtlichen Bedingungen unterliegen können. 